\title{
XXXVI. A table of schocks of earthquake, from September 1839 to the end of 1841, observed at Comrie, near Crieff
}

\section{James Drummond}

To cite this article: James Drummond (1842) XXXVI. A table of schocks of earthquake, from September 1839 to the end of 1841, observed at Comrie, near Crieff , Philosophical Magazine Series 3, 20:130, 240-247, DOI: 10.1080/14786444208650560

To link to this article: http://dx.doi.org/10.1080/14786444208650560

曲 Published online: 01 Jun 2009.

Submit your article to this journal $[\pi$

ЏII Article views: 2

Q View related articles $₫$ 
XXXVI. A Table of Shocks of Earthquake, from September 1839 to the end of 1841, observed at Comrie, near Crieff. By James Drummond, of Comrie*.

CONSIDERING that the theory of the Comrie earthquakes has not yet been satisfactorily established, I thought that an account, by a resident on the spot, of the shocks during the last two years might be of interest, and perhaps of value, to the readers of the Pbilosophical Magazine, and to those engaged in inquiring into these phænomena. It is not my intention to enter into any discussion on the question just now, but I think it right to say that these observations originated in the dissatisfaction I feel as to the theories heretofore propounded, and as to the seat of the disturbance. When I consider that the shocks are only of modern occurrence, that they date only from the opening of quarries into the basaltic dykes on each side of the river Lednoch, and that they are consequent on floods of that river, I cannot feel justified in giving my assent to the doctrine, that they are derived from the percolation of water. Neither can I admit, as is the popular belief here, that the seat of the disturbance is in any of the neighbouring mountains.

I flatter myself, also, that a register of shocks from a resident at Comrie will not be without its uses, as from greater habitude the people of this place are better able to distinguish the shocks.

Before coming to the register, $I$ think it advisable to make a few preliminary observations as to some of the circumstances attendant on the shocks. With regard to 1839 , in which the disturbances were most frequent, both the harvest and winter were, with the exception of very short periods, wet. About eleven on the morning of the 20th of October a drizzling rain came on and continued until seven in the evening of the 23rd, when it cleared up and the stars appeared; neither the sun, moon, nor stars having been seen for about fifty-six hours; at half-past seven, however, it began to rain heavily, and when the shock took place the rain increased. I do not remember that there was any strong wind at the time. On the morning of the 24th it was fair again. It is to be remarked that the rain was general, and the shock was felt over all the central parts of Scotland. It has been said that this shock was felt in all parts at one and the same instant, but I beg leave to demur to the possibility of this, as at Inverness, for instance, the sound was heard some time before the shock, showing that the sound had travelled faster.

* Communicated by the Author. 
In 1841, the end of June and beginning of July were wet nearly every day until the 13th of July, when we had good summer weather. After the shock the weather got cold and stormy, and on the 29th of July we had a regular hurricane from the north-east, doing much damage. After the great shock on the 30th the weather got mild: I observed that the shock was transmitted much further in the direction of the hurricane than in any other.

The greatest shocks happen in summer and harvest. The vertical shocks, I cannot find, are felt further south from the Lednoch quarries than Comrie, on the east further than Tamperran, or on the south-west than Leachkin, being all about half a mile distant. At Kinggart, the nearest house on the north and a mile off, the shocks, vertical at Comrie, \&c., are felt laterally. The greatest of the lateral shocks are nothing in comparison with the greatest of our vertical shocks, and yet the people of Comrie are more afraid of the lateral shocks than of the others. It is considered here that the shocks originate in the mountains, some referring them to Dunira Hill, some to the Cluan, and some to Kinggart. Upon this I will observe, that, on the 12th of October 1839, the people living in those parts observed five shocks, while on the same day at Bruckhill and Saures eight were observed, and at Comrie ten ; on the 23rd following, the people about Dunira and Cluan observed three, while at Comrie some observed fourteen, others eighteen, and some as many as twenty. On the 30th of July, 1840, the Dunira people observed six, and at Comrie fourteen were observed. In general, the shocks are not felt at equal distances in every direction: I think the greatest shocks are felt further north-east and south-west than in any other direction. The shock of the 30th of July, 1841, was not felt at Stirling, twenty-four miles south. Shocks are not felt in every place. Those who are placed on high ground do not observe them near so readily as those on low ground; and they have been felt in coal-mines at Stirling when they have not been felt on the surface. They are not felt alike in Comrie, one house built on a rock not feeling them at all so severely as the others. So slight are the shocks, that to a stranger they are often inappreciable, the ground moving very little with a shock that is felt over a diameter of twelve or twenty-four miles in diameter : it is only those that are felt forty miles and upwards in diameter which cause any alarm. With regard to any external appearance, the only thing I have heard of is from the shepherds watching on Dalengrass, who tell me that they have often observed a thin cloud stretched over the part of the Lednoch valley, which I have assigned as the seat of the disturbance.

Phil. Mag. S. 3. Vol. 20. No. 130. March 1842. R 


\section{M r. J. Drummond's Table of Earthquakes at Comrie.}

To come to the register, $I$ must observe that it gives the state of the river, then the number of what I call gas explosions, next the hour at which the vertical shocks occurred, the number of them, and the utmost distance at which felt; afterwards the lateral shocks, and then the tremours or waving shocks, and lastly the state of the weather. The noises, which I am inclined to consider as arising from gas explosions, are only heard at Comrie, Tamperran and Leachkin, the site of the vertical shocks.

1839.

Sept. 15. Extraordinary flood.

16. Extraordinary flood *.

Oct. 4. 1 gas explosion.

7. 1 gas explosion.

8. 1 gas explosion.

9. 1 gas explosion.

10. I gas explosion. -1 vertical shock at $4 \frac{1}{2}$ a.m., felt 20 miles.-Fair.

11. 1 gas explosion.

12. 5 gas explosions.-1 vertical shock at 1 p.m., felt 14 miles; 1 at $2 \frac{1}{2}$ p.m., felt 8 miles; 1 at 3 p.m., felt 24 miles; 1 at $3 \frac{1}{2}$ p.m., felt 12 miles. -1 tremour at $4 \frac{1}{2}$ p.m., felt 6 miles.-Fair, with clouds.

13. 2 gas explosions.

14. 3 gas explosions.-1 vertical shock at $2 \frac{1}{2}$ a.m., felt 24 miles.

15. 2 gas explosions.

16. 2 gas explosions.- 1 vertical shock at $2 \frac{1}{2}$ a.m., felt 20 miles; 1 at $5 \frac{3}{4}$ p.m., felt 12 miles.-Changeable.

17. 4 gas explosions.

18. 3 gas explosions.

19. 2 gas explosions.

20. 2 gas explosions.

21. 1 gas explosion.

22. 2 gas explosions.

23. High flood. -8 gas explosions. -1 vertical shock at $10 \frac{1}{4}$ p.m.; felt 100 miles.-1 lateral shock at 11 p.m., felt 30 miles; 1 at 12 midnight, felt 10 miles.-Heavy rain.

24. 7 gas explosions. -1 tremour at 5 a.m., felt 6 miles.

25. 2 gas explosions.- -1 tremour at 7 p.m., felt 8 miles.Changeable.

26. 3 gas explosions. -1 tremour at 7 p.m., felt 8 miles.

27. 2 gas explosions.

28. 3 gas explosions.

29. 2 gas explosions.

* The floods throughout the harvest and winter were continual, and sometimes very high. 
Mr. J. Drummond's Table of Earthquakes at Comrie. 243

Oct. 30. 2 gas explosions.

31. 2 gas explosions.

Nov. 1. 1 gas explosion.

2. 2 gas explosions.

3. 1 gas explosion.

4. 1 gas explosion.

5. 2 gas explosions.

6. 1 gas explosion.

7. 1 gas explosion. -1 tremour at 4 a.m., felt 8 miles.Frosty.

8. 2 gas explosions.

9. I gas explosion.

10. 1 gas explosion.

12. 1 gas explosion.

15. 2 gas explosions.

17. 1 gas explosion.

18. 1 gas explosion.

23. 1 gas explosion.

25. 1 gas explosion.

26. 1 gas explosion.

28. 1 gas explosion.

29. 2 gas explosions.

30. 3 gas explosions.

Dec. 1. 1 gas explosion.

2. 2 gas explosions.

3. 1 gas explosion.

4. 1 gas explosion.

5. 2 gas explosions.

6. 1 gas explosion.-1 tremour at 3 a.m., felt 8 miles.Changeable.

7. I gas explosion.

8. 2 gas explosions.

9. 1 gas explosion.

11. 1 gas explosion. -1 tremour at $9 \frac{1}{4}$ p.m., felt 6 miles.

12. 1 gas explosion. -1 tremour at 3 a.m., felt 8 miles.

13. 2 gas explosions.

15. 1 gas explosion.

17. 1 gas explosion.

18. 2 gas explosions.

19. 1 gas explosion.

20. 1 gas explosion.

21. 1 gas explosion.

23. 1 gas explosion.

24. 2 gas explosions.

26. 1 gas explosion.

27. I gas explosion.

28. 2 gas explosions.

30. 1 gas explosion.

31. 1 gas explosion. 
244. Mr. J. Drummond's Table of Earthquakes at Comric.

1840.

Jan. 1. 1 gas explosion.

2. 1 gas explosion.

4. 1 gas explosion.

6. 1 gas explosion.

7. 1 gas explosion.

8. 2 gas explosions.

9. 1 gas explosion.

11. 1 gas explosion.

12. 1 gas explosion.

14. 1 gas explosion.

16. 1 gas explosion.

17. 1 gas explosion.

18. 1 gas explosion.-1 lateral shock at $9 \frac{I}{4}$ p.m., felt 18 miles, - Wind and rain.

19. 2 gas explosions.

20. 1 gas explosion.

23. High floods. - 1 gas explosion.

25. 1 gas explosion.

26. 1 gas explosion.

27. 2 gas explosions. -1 tremour at 6 a.m., felt 5 miles.

28. 2 gas explosions.

31. 1 gas explosion.

Feb. 4. 1 gas explosion.

6. 1 gas explosion.

9. 1 gas explosion.

10. 1 gas explosion. -1 tremour at 4 a.m., felt 6 miles.

12. 1 gas explosion.

14. 1 gas explosion.

16. 1 gas explosion.

18. 1 gas explosion.

20. 1 gas explosion.

22. 1 gas explosion.

24. 1 gas explosion.

25. 1 gas explosion.-1 tremour at 2 p.m., felt 6 miles.

26. 1 gas explosion.

28. 1 gas explosion.

March 7. 1 gas explosion.

8. 2 gas explosions.

9. 1 gas explosion. - 1 tremour at $5 \frac{\mathrm{I}}{2}$ p.m., felt 8 miles.-Fair.

10. 1 gas explosion.

11. 3 gas explosions.

12. 1 gas explosion.

13. 1 gas explosion.-1 tremour at 8 p.m., felt 6 miles.-Fair.

14. 1 gas explosion. --1 tremour at $9 \frac{1}{2}$ p.m., felt 6 miles. - Fair.

$13,14,15,16$, Lednoch flooded by the melting of snow on the North hills.

21. 1 gas explosion.

23. 1 gas explosion.

24. 1 gas explosion. 
Mr. J. Drummond's Table of Earthquakes at Comrie. 245

March 25. 1 gas explosion.

26. 1 gas explosion.

27. 1 gas explosion.

Apri] 1. 2 gas explosions.--Slight snow.

3. 1 gas explosion.

5. 1 gas explosion.

7. 2 gas explosions.-1 lateral shock at 4 p.m., felt 12 miles. -Dry and cloudy.

10. 1 gas explosion.

12. 1 gas explosion.

13. 1 gas explosion.

May 19. 2 gas explosions.- -1 tremour at $1 \frac{3}{4}$ p.m., felt 6 miles.Dry and cloudy.

22. I gas explosion.

25.

29. I gas explosion.

16. Flood.-1 gas explosion.

[Apparently there is some error

25. Flood.

July 2. 2 gas explosions.

15. 2 gas explosions.

17. 1 gas explosion.

19. Flood.

21. Flood.

25. 1 gas explosion.

August 6. I gas explosion.

17. Flood.

Sept. 1. 1 gas explosion.

19. 2 gas explosions.

23. Flood.

27. 1 gas explosion.

28. Flood.

21. 1 gas explosion.

26. 1 gas explosion.-1 vertical shock at 7 p.m., felt 12 miles. -Mizzly rain.

27. 1 gas explosion.

30. Flood.

Nov. 4. Slight flood.

6. Slight flood.

12. 2 gas explosions.

13. 3 gas explosions.

14. 1 gas explosion.

16. Slight flood.-1 gas explosion.

17. 2 gas explosions.

Dec. 7. High flood.

13. ? gas explosions.

15. Flood. -1 gas explosion.

24. 2 gas explosions.

25. 1 gas explosion.

1841 .

Jan. 6. 1 gas explosion. -1 tremour at 12 midnight, felt 6 miles. - Frosty. 
246 Mr. J. Drummond's Table of Earthquakes at Comrie.

Jan. 10. 2 gas explosions.

15. 1 gas explosion.

17. 2 gas explosions.

22. Flood.

27. Flood.

Feb. 1. 1 gas explosion.

13. Flood.

14. Flood.-1 gas explosion.

15. Flood.

16. 1 gas explosion.

March 6. 1 gas explosion.

10. 1 lateral shock at 5 p.m., felt 15 miles.-Dry and cloudy.

19. Flood.

21 and 22. Floods. -2 gas explosions. -1 tremour at $6 \frac{\mathrm{I}}{2}$ a.m., felt 6 miles.-Fair.

23 and 30. Flood.

April 3. 1 gas explosion.

9. 1 vertical shock at 8 a.m., felt 10 miles.

14. 1 tremour at 5 a.m., felt 5 miles.

24. 1 vertical shock at $1 \frac{3}{4}$ p.m., felt 10 miles.

26 and 27 . Slight floods.

May 3, 4 and 10. Slight floods.

5. 1 gas explosion.

22. 1 tremour at 12 noon, felt 6 miles.

26. 1 gas explosion.

27. 1 gas explosion.

28. 1 gas explosion.

30. 1 tremour at 7 a.m., felt 5 miles:

June 21, 23 and 28. Floods.

July 2. 1 gas explosion.

10, 11 and 12. Slight floods.

23. I gas explosion. - 1 lateral shock at $4 \frac{1}{4}$ p.m., felt 10 miles. -Mild and warm.

25.

26. 1 gas explosion.

27. 1 gas explosion.

30. 12 gas explosions.- 1 vertical shock at 8 a.m., felt 10 miles: I at 2 p.m., felt 60 miles.-Dry, cold and cloudy.

31. 1 gas explosion. $\rightarrow 1$ vertical shock at 8 a.m., felt 8 miles. -Dry, cold and cloudy.

August 1. 1 gas explosion.

2. 1 gas explosion.

3. 1 gas explosion.

5. Flood.

7. 1 gas explosion.

8. Flood.

10. 1 gas explosion.

12. 1 vertical shock at 10 a.m., felt 10 miles,-Fair.

14. Flood.

19. High flood.

22. 1 gas explosion. 
Mr. J. Drummond's Table of Earthquakes at Comrie. 247

Aug. 23. High flood.

30. Flood.

Sept. 1. 1 gas explosion.

2, 9 and 10. Slight flood.

10. 1 vertical shock at $11 \frac{\mathrm{I}}{4} \mathrm{p} . \mathrm{m}$., felt 20 miles.-Changeable, dry and fair after the shock, and starlight at the time.

11. I vertical shock at $2 \frac{\mathrm{I}}{4}$ a.m., felt 15 miles.-Fair; rain immediately after the shock.

15. High flood.

16. I gas explosion

22. 1 gas explosion.

23. 1 gas explosion. -1 vertical shock at $1 \frac{1}{4}$ a.m., felt 10 miles.-Changeable.

24. Flood.

25. Flood.-1 gas explosion.-1 lateral shock at 9 p.m., felt 8 miles.-Rain.

27, 28 and 29. Floods.

Oct. 10, 13, 14, 17 and 20. High floods.

13. 1 tremour at 12 midnight, felt 6 miles.-Fair.

23. Slight flood.-1 lateral shock at 1 a.m., felt 8 miles.Rainy.

Nov. 3. Flood.-3 gas explosions.

5. 1 tremour at 1 a.m., felt 5 miles.-Fair.

6. 1 gas explosion. -1 tremour at 8 a.m., felt 6 miles.Changeable.

8. 1 gas explosion. - 1 tremour at 8 a.nu., felt 5 miles.-Fair.

18. I gas explosion.-1 tremour at $8 \frac{1}{2}$ a.m., felt 5 miles.-Frosty.

29. Slight flood.

Dec. 1. Flood.

3. Slight flood.-1 gas explosion.

6. Slight flood.-1 tremour at 3 a.m., felt 6 miles. - Rain.

7. 1 gas explosion.

12. Flood.

15. High flood.

19. 1 vertical shock at $1 \frac{x}{2}$ a.m., felt 5 miles.-Frosty.

I am aware that some persons who have been keeping a list of shocks reject the very small sounds, and mark the louder ones as slight shocks of earthquakes, whether those sounds be occasioned by the explosions of hydrogen and oxygen gas or not: it is contrary to common sense to call a slight sound a shock of an earthquake. Again, it is to be observed that the people at Comrie were in constant terror during the latter part of 1839 , and it is possible that people imagined they heard sounds of earthquakes when there were none: making due allowance for this, it is certain that the sounds were uncommonly frequent during that year (1839).

James Drummond. 